\section{An inter-comparison of isotopic com- position of neon via chemical assays and thermal analyses (IUPAC Technical Report)}

Steur, P., Yang, I., Kim, J., et al.

Pure and Applied Chemistry, 2019

Volume 91, Issue 11, pp. 1869-1882

https://doi.org/10.1515/pac-2017-1203

From 2003 to 2014, a study on the effect of isotopic composition on the triple point temperature of neon was conducted under the framework of a project involving laboratories from 11 countries. Natural neon from commercial sources of different isotopic composition, high-purity ${ }^{20} \mathrm{Ne}$ and ${ }^{22} \mathrm{Ne}$ isotopes, and certified artificial isotopic mixtures were used. The thermometric studies comprised: a) a total of 131 analytical assays from three laboratories on the isotopic composition of samples taken from 31 different bottles of neon with chemical gas purity $99.99 \mathrm{~mol} \%$ to $99.9995+\mathrm{mol} \%$, including chemical impurities for some samples, with up to 12 assays per sample; b) multi-laboratory thermal analyses, with accuracies ranging up to better than $50 \mu \mathrm{K}(k \approx 2)$, on 39 samples, almost all permanently sealed in metal cells, for the determination of the liquidus-point temperature of the triple point as a function of isotopic composition. The thermometric studies also constitute an international inter-comparison of thermal and analytical assays on the isotopic composition-and occasionally of the chemical impurities-of neon. These tests are critically needed for top-accuracy thermometry.

The main results of the inter-comparison of the various chemical assays, and of the comparisons between the assays and the results of thermal analyses,

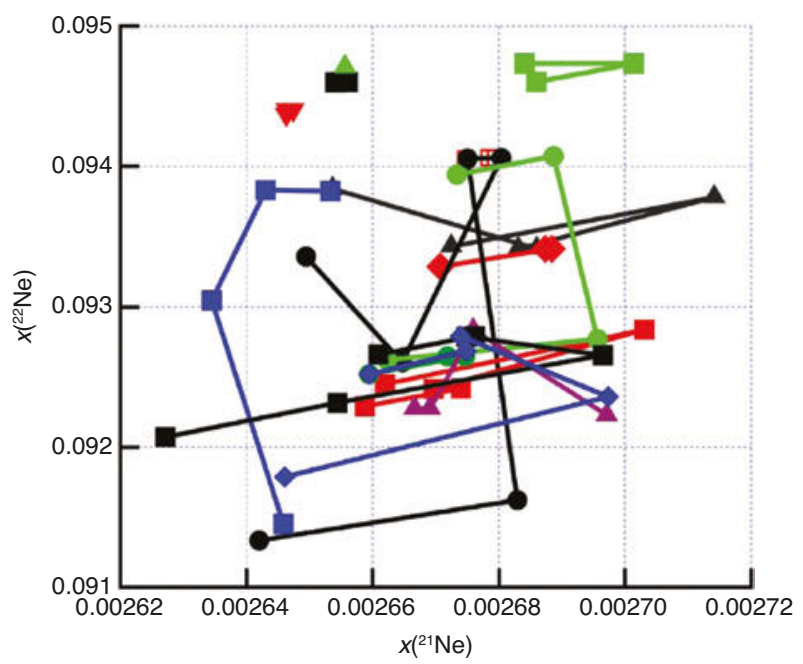

are reported. They show discrepancies in $x\left({ }^{20,21,22} \mathrm{Ne}\right)$, especially for $x\left({ }^{22} \mathrm{Ne}\right)$, in 'natural' neon, for the same gas bottle, equivalent to an uncertainty of up to 165 $\mu \mathrm{K}(k=1)$ in the triple point temperature, as measured by all testing laboratories, and of about $100 \mu \mathrm{K}(k=1)$ as measured from a single testing laboratory. This is an unsatisfactory situation for thermometry, since it is difficult to obtain a reliable and accurate isotopic assay for neon, thus limiting the accuracy of the realisation of the neon triple point temperature as a ITS-90 reference point to well above $50 \mu \mathrm{K}$. However, it also discloses a strong limitation in the relevant analytical chemistry.

\section{Definition of the chalcogen bond (IUPAC Recommendations 2019)}

Aakeroy, C., Bryce, D., Desiraju, G., et al.

Pure and Applied Chemistry, 2019

Volume 91, Issue 11, pp. 1889-1892

https://doi.org/10.1515/pac-2018-0713

This recommendation proposes a definition for the term "chalcogen bond"; it is recommended that the term is used to designate the specific subset of inter- and intramolecular interactions formed by chalcogen atoms wherein the Group 16 element is the electrophilic site.

\section{chalcogen bond (ChB)}

net attractive interaction between an electrophilic region associated with a chalcogen atom in a molecular entity and a nucleophilic region in another, or the same, molecular entity.

Critically evaluated propagation rate coefficients for radical polymerizations: acrylates and vinyl acetate in bulk (IUPAC Technical Report)

Hutchinson, R. and Beuermann, S. Pure and Applied Chemistry, 2019 Volume 91, Issue 11, pp. 1883-1888 https://doi.org/10.1515/pac-2018-1108

Arrhenius parameters capturing the temperature dependence of radical polymerization propagation rate coefficients, $k_{p}$, for methyl acrylate, butyl acrylate, and vinyl acetate in bulk are reported, based on the fitting of benchmark data sets compiled from independent 